\title{
Protée
}

\section{Manger le livre. Désémiotisation et imaginaire de la fin}

\section{Bertrand Gervais}

Volume 27, numéro 3, 1999

L'imaginaire de la fin

URI : https://id.erudit.org/iderudit/030567ar

DOI : https://doi.org/10.7202/030567ar

Aller au sommaire du numéro

Éditeur(s)

Département des arts et lettres - Université du Québec à Chicoutimi

ISSN

0300-3523 (imprimé)

1708-2307 (numérique)

Découvrir la revue

Citer cet article

Gervais, B. (1999). Manger le livre. Désémiotisation et imaginaire de la fin. Protée, 27(3), 7-18. https://doi.org/10.7202/030567ar

\section{Résumé de l'article}

Un des traits de l'imaginaire de la fin, outre les scénarios apocalyptiques, est une langue devenue opaque, à la limite d'être une chose, ou alors une langue démultipliée, aux capacités illimités. Le chronotope de la fin provoque ce qu'on peut appeler une désémiotisation de la langue, liée à un déséquilibre des interprétants, par lesquels sont opérées les attributions de sens. Pour en rendre compte, l'auteur donne trois exemples, dont le plus important est tiré de l'Apocalypse de Jean. C'est la scène où l'Ange offre à Jean de manger un livre. Comment expliquer un tel geste ? Quelles en sont les conséquences ? L'auteur compare cette scène à un récit de Maurice Blanchot (Le Dernier Mot) et à la dernière scène du roman City of Glass de Paul Auster. 


\section{AN G ER LE LIVRE D ÉSÉMIOTISATION ET IMAGINAIRE DE LA FIN}
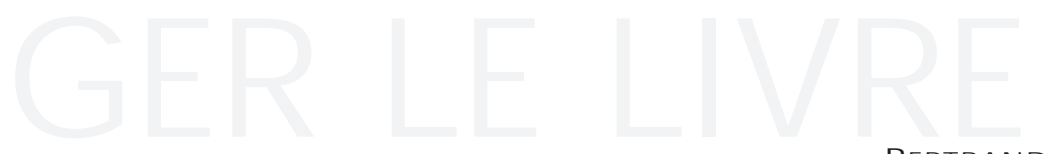

BERTRAND GERVAIS

L'apôtre nous dit qu'au commencement était le verbe. Il ne nous donne aucune assurance quant à la fin.

George Steiner

Dans «Le dernier mot", récit de Maurice Blanchot tiré d'Après coup (1983), la confusion annonciatrice de la fin du monde ne fait pas qu'atteindre le corps social ou même les sujets qui doivent se déprendre de ses rets, elle s'étend au langage luimême qui devient opaque et étranger. La langue n'y joue plus son rôle usuel, elle s'écrase en quelque sorte, devenant une masse concrète, lourde et encombrante. Les mots commencent à ne plus rien dire, la transparence qui est le gage de leur fonctionnalité s'évapore, et c'est leur matière même qui réapparaît et s'impose, avec d'autant plus de force qu'elle en marque l'inutilité.

Le narrateur se promène dans les rues de la ville et, dès les premiers instants, il remarque que les paroles qu'il entend sonnent mal à ses oreilles, comme si les mots avaient été détournés de leur cours naturel (1983: 57 et 60). Le vacarme est grand et, de rue en rue, il se promène au milieu de débris d'une fête que rien n'explique, d'une fête qui marque le retrait de toute forme de loi.

À certains carrefours la terre tremblait et il semblait que la populace marchât sur le vide, qu'elle franchissait sur une passerelle de vociférations. La grande consécration du jusqu'à ce que eut lieu vers midi. Avec des débris de paroles, comme si du langage n'eussent subsisté que les formes d'une longue phrase écrasée par le piétinement de la foule, on modula le chant d'un mot qui transparaissait à travers n'importe quel hurlement. (1983: 63)

Cette métaphore littéralisée témoigne d'une parole détraquée, d'un accès au langage qui ne répond plus aux interprétants habituels. Les mots paraissent marqués d'une inquiétante étrangeté, d'une altérité irréductible. Des contenants vidés de leur contenu, ou pour mieux dire désémiotisés.

Le texte se déploie selon une logique de l'excès, où se côtoient, à une extrémité du spectre, un «idéal antérieur au langage» (1983: 70), une langue présente «dans un état d'innocence que ne serait pas venue troubler la recherche du premier mot» 
(1983: 71), une langue primordiale qui résiste aux perceptions et, à l'autre extrémité, une langue réduite à l'état de ruines, faite de mots brisés, qui ne servent plus à communiquer (1983: 65), un code dont le principe identitaire a été éliminé.

Le texte cherche à poser ce qu'il en serait du dernier mot, d'un mot qui viendrait sceller le sort des mots, qui en dirait par conséquent l'ultime état avant sa disparition. Ce moment fugitif, Blanchot le décrit avec précision:

Jusqu'au dernier moment, je vais être tenté d'ajouter un mot à ce qui a été dit. Mais pourquoi un mot serait-il le dernier? La dernière parole, ce n'est déjà plus une parole et, cependant, ce n'est pas le commencement d'autre chose. Je vous demande donc de vous rappeler ceci, pour bien conduire vos observations: le dernier mot ne peut être un mot, ni l'absence de mot, ni autre chose qu'un mot. (1983: 77)

Ce dernier mot, dénudé de tout ce qui a pu le constituer comme mot, de ce qui a pu en faire plus qu'une simple chose, rabattu par conséquent à une matérialité redevenue prépondérante, je fais ici l'hypothèse qu'il constitue une caractéristique des imaginaires de la fin, au même titre que les désordres de toutes sortes, le chaos politique et social, les catastrophes et les jugements. Le chronotope de la fin est marqué par une désémiotisation de la langue, par une perte graduelle des mécanismes de signification. La désémiotisation rend compte de ces situations où l'opacité du signe est devenue prépondérante, éliminant de fait toute possibilité de renvoi à des objets de pensée. Les attributions se dénouent, les renvois ne se font plus et il ne reste, des signes, qu'une matérialité énigmatique ${ }^{1}$.

\section{MANGER LE LIVRE}

La parole prophétique est lourde. Maurice Blanchot

Cette adéquation entre imaginaire de la fin et désémiotisation est présente dès les textes fondateurs de la pensée apocalyptique, et de façon plus précise

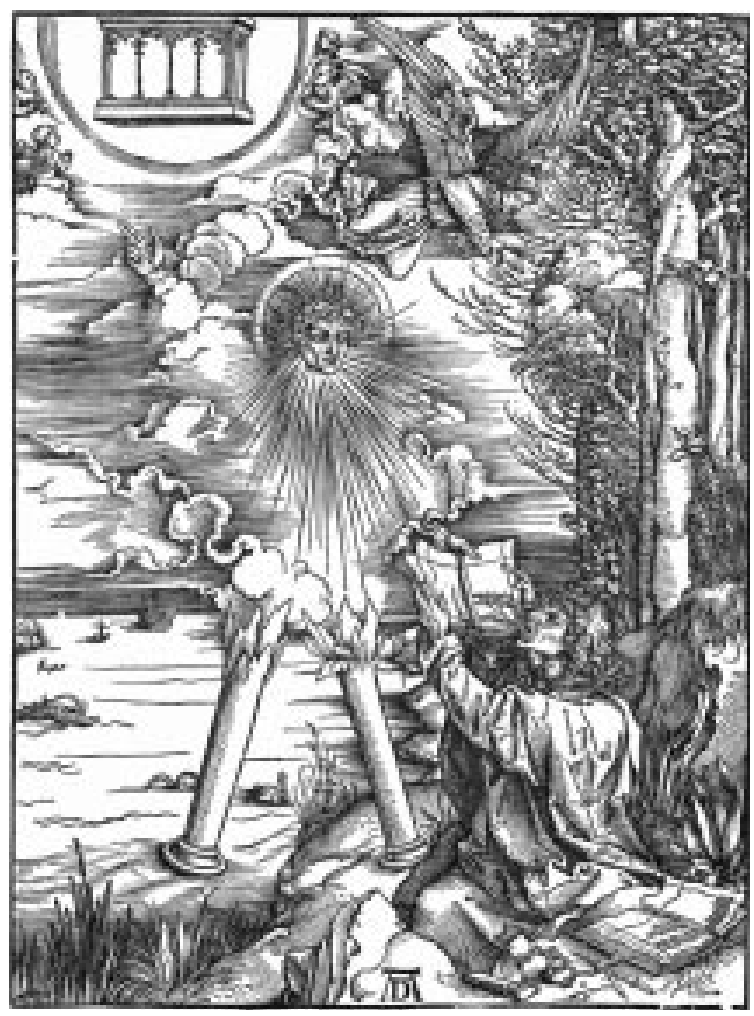

Gravure d'Albrecht Dürer

dans l'Apocalypse de Jean ${ }^{2}$. Non pas que l'équivalence y soit formulée de façon explicite ou formelle, mais on $\mathrm{y}$ retrouve une scène qui repose sur une même matérialité de la langue à l'approche de la fin. J'aimerais en faire une description détaillée.

La scène en question apparaît, dans l'Apocalypse, juste avant que la septième trompette ne résonne, au chapitre dix de la prophétie. Tous les sceaux ont été ouverts, libérant des symboles mystérieux, et les malheurs ont commencé à s'abattre sur le monde: grêle et feu mêlés de sang, les eaux devenues amères et empoisonnées, la lune et les étoiles frappées de ténèbres, une invasion de sauterelles, etc. Jean voit alors un Ange descendre du ciel enveloppé d'une nuée, tenant «en sa main un petit livre ouvert» (10: 2). L'Ange pose le pied droit sur la mer, le gauche sur la terre, et il pousse une puissante clameur pareille au rugissement du lion. Après quoi, les sept tonnerres font retentir leur voix $(10: 2-3)$. 
Que disent les sept tonnerres? Jean ne le révèle pas. Mais assurément il le sait, car il entreprend aussitôt d'écrire et de transcrire ce qui a été dit. Jean peut décoder le tonnerre comme il parvient à lire à travers le rugissement du lion. Si pour nous, lecteurs, ces paroles apparaissent déjà comme des signes inaccessibles, des manifestations de la nature sans autre signification que celle, générique, qui permet de les identifier comme tonnerre et rugissement, Jean peut, lui, les comprendre. Mais l'Ange ne le laisse pas les transcrire. Avant que ne sonne la septième et dernière trompette, il lui dit: "Tiens secrètes les paroles des sept tonnerres et ne les écris pas» (10:5). Puis il déclare:

"Plus de délai! Mais au jour où l'on entendra le septième Ange, quand il sonnera de la trompette, alors sera consommé le mystère de Dieu, selon la bonne nouvelle qu'il en a donnée à ses serviteurs les prophètes». Puis la voix du ciel, que j'avais entendue, me parla de nouveau: "Va prendre le petit livre ouvert dans la main de l'Ange debout sur la mer et sur la terre». Je m'en fus alors prier l'Ange de me donner le petit livre; et lui me dit: "Tiens, mange-le; il te remplira les entrailles d'amertume, mais en ta bouche il aura la douceur du miel». Je pris le petit livre de la main de l'Ange et l'avalai; dans ma bouche, il avait la douceur du miel, mais quand je l'eus mangé, il remplit mes entrailles d'amertume. Alors on me dit: "Il te faut de nouveau prophétiser contre une foule de peuples, de nations, de langues et de rois». (10:6-11)

Quel est ce petit livre qu'avale Jean? À quoi correspond cette eucharistie? Car il y a là, en toutes lettres, un acte de communion avec Dieu. Sans vouloir à tout prix prendre au pied de la lettre cette scène de lecture dont la dimension allégorique est indiscutable, on peut se demander comment Jean parvient à ingurgiter ce livre, si petit soit-il. Comment avale-t-on un codex? À quelle logique ce geste répondil? Qu'est-ce qui le motive?

Disons immédiatement que de nombreuses représentations picturales sont venues inscrire ce geste comme un motif. Dans la tenture du château d'Angers, réalisée à la fin du XIVe siècle et qui dépeint en plus de quatre-vingts tableaux l'Apocalypse, on voit, face à l'Ange, Jean debout qui tient deux livres ouverts. Un premier de la main droite et un second, de la gauche, qu'il prend entre ses dents, par son coin supérieur droit (à moins que le livre ne soit inversé). Dans son illustration de l'Apocalypse, à la fin du XVI siècle, Albrecht Dürer en a donné une version similaire, où l'on voit Jean, à genoux cette fois, recevoir un livre ouvert de l'Ange et en mettre le coin inférieur droit dans sa bouche, livre dont les pages semblent froissées par cette consommation déjà amorcée (voir page ci-contre). Le codex donne l'impression de connaître un début d'anamorphose, comme si sa masse pouvait ainsi s'étirer jusqu'à glisser dans le fond de la gorge. Les illustrations de Durër paraissent à ce point définitives qu'un historien d'art a affirmé que "Toutes les Apocalypses du XVIe siècle dérivent, directement ou par des intermédiaires, de l'Apocalypse de Durër» (Mâle, 1925: 443). Alberto Manguel reproduit d'ailleurs une illustration russe du XVII e siècle, qui est une reprise à peine déguisée de la gravure de Dürer (1996: 172).

L'image, reproduite avec régularité, est à ce point ancrée dans l'imaginaire occidental que dans un roman comme Le Nom de la rose, d'Umberto Eco, qui se tisse sur la trame de l'Apocalypse, une des scènes finales se sert du motif du livre mangé. Le codex n'y est pas mangé d'un coup, le livre entier saisi par un de ses coins, il est au contraire absorbé page par page, dans une représentation devenue réaliste, répondant par conséquent aux critères du vraisemblable. On y apprend ainsi qu'un des personnages commence «de ses mains décharnées et diaphanes à déchirer, par morceaux et par bandes, les pages molles du manuscrit, se les déposant en lambeaux dans la bouche, et mâchant lentement comme s'il consommait l'hostie et voulait la faire chair de sa propre chair» (1982: 485). La référence au texte de l'Apocalypse est explicite ${ }^{3}$.

Pour tenter de comprendre ce geste et ses conséquences en termes de désémiotisation, il semble important de cerner d'abord son contexte et ses déterminations. Ainsi, qu'en est-il du livre qui est lu? Est-ce bel et bien un codex qui est avalé, un livre avec 
des pages? Et pourquoi ne peut-il être lu? En fait, qu'entend-on par lire au début de l'ère chrétienne?

Manger le livre. Nos conceptions contemporaines du livre tendent à accréditer l'absorption d'un codex, impression laissée entre autres par les traductions contemporaines de la Bible. Pourtant, il semble plus juste de dire que ce n'est pas un codex, mais bel et bien un volumen qui est avalé. Un rouleau, tel qu'on les possédait et les lisait à cette époque.

Les deux formes de livre coexistent en fait au début de l'ère chrétienne, et plus précisément au premier siècle, époque de la rédaction de l'Apocalypse. Le codex est déjà apparu mais son usage ne s'est pas encore répandu. Il le sera très rapidement, le codex devenant à partir du II ${ }^{e}$ siècle après J.-C. la forme préférée pour les textes chrétiens. Et il s'impose rapidement en raison de son moindre coût, de l'usage du parchemin plutôt que du papyrus, de sa forme pratique qui, non seulement laisse le lecteur plus libre de ses mouvements et facilite l'étude et une consultation en profondeur (Cavallo et Chartier, 1997: 23; Saenger, 1982 : 374), mais encore, du fait de son format, se dissimule bien dans les vêtements et se transporte facilement (Manguel, 1996: 48). L'habitude de penser le livre sous forme de codex se prend donc rapidement, ce qui explique peut-être les habitudes interprétatives actuelles, qui ne parviennent pas à se détacher de ce format.

Ces habitudes ne sont pas sans leurs points aveugles. Ainsi la traduction de l'École biblique de Jérusalem use d'une formule étonnante en regard de ce qui vient d'être décrit. Au chapitre six, il est dit que "le ciel disparut comme un livre qu'on roule» (6:14). Ce qui est ouvert après la sixième trompette peut se dérouler à la rupture du sixième sceau, dans un croisement médiologique inopiné 4 . Par contre, des entreprises de traduction qui cherchent à se dégager des habitudes interprétatives actuelles et à respecter les textes d'origine parlent quant à elles d'un volume mangé par Jean. Dans leur traduction de l'Apocalypse, Chouraqui choisit le terme même de volume (1982: 501) et Tresmontant mentionne, pour sa part, un rouleau, un petit rouleau (1984: 39).
Quant à la lecture au début de l'ère chrétienne, il faut là encore se départir de ces conceptions contemporaines d'une lecture silencieuse et extensive pour reconnaitre que la forme principale de lecture de l'époque se faisait à haute voix. Les textes étaient écrits en scriptio continua, c'est-à-dire sans séparation entre les mots, et par conséquent sans aucune ponctuation, sans distinction entre les majuscules et les minuscules, une écriture qui restait «inintelligible et inerte sans énonciation à voix haute" (Cavallo et Chartier, 1997: 14; Saenger, 1982: 372 et passim). Et il en va de même a fortiori en hébreu, où les voyelles ne sont pas marquées, de sorte qu'il faut lire à voix haute le texte pour en inférer son sens. L'acte de lire, dans la culture hébraïque, est un acte de parole; lire et dire sont même désignés par un même verbe (Manguel, 1996: 45; chose et parole sont de plus désignées par le même mot, "davar»). Dans un tel contexte culturel, donner un livre à lire aurait impliqué, d'une part, de forcer Jean à un acte long et laborieux de déchiffrement du texte écrit et, d'autre part, de l'inciter à une oralisation, à une énonciation à voix haute ("Tiens secrètes les paroles des sept tonnerres" (10: 4), avait dit l'ange précédemment). Le manger suppose au contraire un acte beaucoup plus immédiat - si tant est qu'un rouleau puisse être avalé d'une traite-, où Jean peut à la fois prendre connaissance du texte, sans avoir à révéler son contenu à voix haute, et, plus important encore, ne pas avoir à poser le regard sur Dieu, par l'entremise de sa parole, tout en la recevant.

Le contexte culturel général de cet acte permet donc d'en comprendre certaines déterminations. Mais, pour en saisir toute la signification, il faut le replacer dans le cadre plus étroit de l'univers biblique. Ainsi, cette scène n'est pas la première, dans la Bible, où un livre est mangé, où un savoir est acquis par des voies inhabituelles et permet l'apostolat. Ézéchiel, dans sa prophétie, avait déjà décrit une situation à peu de chose près identique. Le prophète entend la parole de Yahvé qui lui demande: «Ouvre la bouche et mange ce que je vais te donner» (Ézé, 2: 8). Ézéchiel voit ensuite une main qui tient un «volume 
roulé», où il est écrit au recto et au verso (fait inhabituel, les volumes ne portent de l'écriture que sur un côté). L'ordre est explicite:

"Fils d'homme, ce qui t'est présenté, mange-le; mange ce volume et va parler à la maison d'Israël». J'ouvris la bouche et il me fit manger ce volume, puis il me dit: "Fils d'homme, nourris-toi et rassasie-toi de ce volume que je te donne». Je le mangeai et, dans ma bouche, il fut doux comme du miel. (Ézé, 3: 1-3)

L'absorption du livre donne à Ézéchiel un savoir qui lui permet de porter les paroles de Yahvé au peuple même d'Israël, réfractaire aux lois de son Dieu. La scène est identique à celle présente chez Jean, à ceci près que ce dernier, dans sa reprise, y ajoute l'amertume ${ }^{5}$. Le volume pour Ézéchiel est uniquement doux comme le miel. Il y manque l'opposition qui devient explicite dans l'Apocalypse, où le volume se trouve à la fois être sucré et amer, soit deux des goûts fondamentaux (avec l'acide et le salé). Ce qui est écrit à l'encre, ce qui est présent en bouche, sur la langue et le palais, relève du sucré et de l'agréable; et ce qui est révélé, dès que l'estomac décompose le tout, ce qui est contenu dans le livre est amer, pénible et cruel. Le miel dit la bonté de Dieu pour son prophète, et l'amertume dit aussi sa colère pour ceux qui n'ont pas encore accepté sa parole. Dans l'Apocalypse, cette amertume est aussi celle de l'Absinthe, l'astre qui tombe sur le tiers des fleuves et sur les sources et qui rend les eaux amères $(8: 11)$.

L'absorption du livre transmet au prophète un double savoir, un contenu mais aussi un don, celui de la parole. Digérer le livre permet de prophétiser, d'en dire plus et, surtout, du moins chez Jean, d'aller audelà des langues (Apo, 10: 11). Les conséquences ressemblent à celles du miracle de la Pentecôte. Dans les Actes des Apôtres, il est raconté que ceux-ci étaient réunis quand retentit du ciel un terrible fracas et qu'apparaissent des langues de feu qui viennent se poser sur chacun des apôtres: "Tous furent alors remplis de l'Esprit Saint et commencèrent à parler en d'autres langues, selon que l'Esprit leur donnait de s'exprimer " (Act 2: 3-4). Des dévots de toutes les nations sont présents et chacun entend parler dans son propre idiome, comme si ce que les apôtres parlaient était une langue universelle, un langage d'avant les langues, qui permet par conséquent de toutes les recouvrir. L'exploit a ceci de surprenant qu'il est lié, dans les Actes des Apôtres, aux jours du Jugement dernier. Aux dévots qui ironisent et qui accusent les apôtres d'être pleins de vin doux, Pierre répond que ces gens ne sont pas ivres, mais qu'ils confirment ce que le prophète Joël a dit: «Il se fera dans les derniers jours, dit Dieu, que je répandrai de mon Esprit sur toute chair. Alors vos fils et vos filles prophétiseront $[\ldots] »(\text { Act } 2: 17)^{6}$.

L'apparition d'une langue plurielle et transcendante signale clairement un jugement dernier, une intervention de Dieu, dont la présence se fait sentir de façon non équivoque. On se doit de remarquer, à ce titre, la très grande clôture de la Bible. Cela commence et se termine de la même façon. Et la Genèse et l'Apocalypse, les deux limites de ce texte fondateur de la chrétienté, sont en effet marquées par une langue transcendante. À la langue démultipliée de Jean, voisine dans ses possibilités de la langue qui prévalait avant la chute de la tour de Babel, correspond la langue adamique. Dans la Genèse, Dieu modèle du sol tous les animaux et il les amène à Adam pour voir comment celui-ci les appellera: «chacun devait porter le nom que l'homme lui aurait donné» (Gen 2: 19). Le savoir du premier homme est inné et sa langue, les noms qu'il donne aux animaux sont ceux que Dieu leur reconnaîtra. L'homme et Dieu parlent la même langue. Une langue qui est dans un rapport nécessaire aux choses de ce monde, puisqu'elle permet de les identifier exactement, hors de toute arbitrarité. Et c'est bien, en quelque sorte, la langue qui est récupérée par Jean, en mangeant le livre, et qui lui permet de prophétiser auprès de peuples, de nations et de langues qu'il ne connaît pas a priori. De l'arbitraire et du contingent, il est retourné au nécessaire et au motivé.

Savoir et nourriture sont associés dans un autre passage de la Genèse, l'événement à l'origine de l'expulsion du jardin d'Éden. Ėve et Adam mangent 
le fruit défendu de l'arbre de la connaissance du bien et du mal, l'arbre du discernement. Et, de fait, à peine ont-ils avalé ce fruit que leurs yeux s'ouvrent. Leur propre nudité apparaît sans détour et ils s'empressent d'y remédier, en cousant des feuilles de figuier pour s'en faire des pagnes. Ils savent reconnaître le bien et le mal, ce que Dieu comprend aussitôt. Le fruit, l'aliment absorbé et digéré est source de savoir, de la même façon que le livre l'est pour Jean, le volumen fait de papyrus, d'une matière végétale. Mais, tandis que dans le premier cas, le savoir obtenu provoque l'ire de Dieu, et la rupture, dans le second, il signale l'alliance et l'exemption. Il inscrit Jean du côté des élus.

Le livre mangé réunit en fait, à lui seul, les deux polarités du dysfonctionnement langagier des imaginaires de la fin. Dysfonctionnement déjà noté dans le récit de Blanchot. Il est une matérialité exacerbée, une langue réduite à ses aspects concrets, qui circonviennent les composantes habituelles des processus sémiotiques, se donnant à manger plutôt qu'à entendre ou à lire. Et il donne accès à un savoir et à une langue aux possibilités infinies, c'est-à-dire à une incomparable sémiotisation, qui va jusqu'à défier le sémiotique. Les interprétants (au sens peircien, $c f$. Deladalle, 1979; Peirce, 1978) sont instables: ou ils s'évanouissent pour ne plus laisser que des representamens amputés de ce qui les définit habituellement comme signes; ou ils s'imposent comme interprétants universels, rendant les representamens superflus, car les attributions se font indépendamment des signes-choses qui en sont la partie première. Évanouis, nous avons un livre qui se mange au lieu de se lire; omnipotents, nous avons un prophète qui parle toutes les langues, qui parle indépendamment des representamens, puisqu'ils sont devenus interchangeables. Une langue sans plus de loi, autre que physique, celle du corps et de son système digestif; une langue qui n'est plus que loi, dont la portée englobe l'humanité entière. Un token qui a perdu son rapport au type; un type qui n'a plus besoin de token pour exister.

\section{L'INTENSIFICATION SÉMIOTIQUE}

La Révélation est perçue comme une voix et contemplée dans l'image.

Elle est divisée tout d'abord par les sens, puis lorsqu'elle est départie.

Le Voyant traverse le rideau qui sépare le divisé de l'indivis.

Ernst Jünger

Si la désémiotisation des langues est une marque des imaginaires de la fin, son contrepoint nécessaire l'est aussi, c'est-à-dire une intensification de l'activité sémiotique. Les deux agissent de façon proportionnelle. Plus le monde se fait hermétique, et le langage opaque, et plus le besoin de le comprendre est grand, plus il devient impérieux d'interpréter les signes par lesquels il se fait connaître. C'est le principe des sémiotiques hermétiques, décrites par Umberto Eco (1992). Dans l'Apocalypse, cette intensification prend la forme d'une pléthore de situations de communication et d'une surabondance de signes d'autant plus attirants qu'ils demeurent mystérieux. Le texte est fait de perceptions à comprendre, de paroles à interpréter, d'énigmes à résoudre, d'écrits à lire et à digérer, d'une parole prophétique à transmettre.

Le texte est ainsi irisé de gestes de communication. Jean nous transmet ce qu'il voit et entend, sans jamais effacer les traces de cette vision et de cette écoute. Les événements révélés nous sont communiqués constamment médiatisés par le prophète. Il est l'intermédiaire, le truchement par lequel les faits seront connus. Des choses apparaissent ainsi à ses yeux, on lui montre des merveilles, il affirme constamment que ses visions se poursuivent $(5: 11$; $6: 1,12 ; 8: 13 ; 15: 5)$, visions d'êtres fabuleux, d'Anges descendus du Ciel, de malheurs et de fléaux. Ces images répondent aux voix qu'il entend et qui l'informent du sens des événements, de la signification des choses et des êtres ${ }^{7}$. Puis, il lui est demandé d'écrire ce qu'il a vu $(1: 19)$, de transcrire ce qu'il a entendu $(2: 1,8,12,18 ; 3: 1,7,14)$. Les visions et paroles se multiplient et se complètent pour constituer un spectacle irréfutable. Il voit des âmes et des Anges qui hurlent d'une voix puissante, il entend crier et des êtres apparaissent à ses yeux. On proclame, on 
divulgue, on publie; des trompettes sonnent, des voix clament; il entend des bruits; les tonnerres parlent.

Des Anges viennent et le transportent en esprit dans des lieux étrangers. Ces visions sont narrativisées et elles s'articulent pour former une séquence complexe, avec ses étapes et ses retournements ${ }^{8}$.

Le caractère incroyable de ces visions est marqué de plus par des approximations, des métaphores et des comparaisons. Ces approximations révèlent l'étrangeté des phénomènes et l'incapacité de Jean de les décrire de façon littérale. Ce ne sont pas des voix qui lui parlent, ce sont des bruits qui sont "comme une voix" (6:6), ou une voix qui clame comme une trompette (1: 10), une «voix forte comme rugit le lion» $(10: 3)$. Ces mots qui ne suffisent pas apparaissent dès les premiers chapitres, quand la vision commence. Jean voit un être

[...] comme un Fils d'homme [...] Sa tête, avec ses cheveux blancs, est comme de la laine blanche, comme de la neige, ses yeux comme une flamme ardente, ses pieds pareils à de l'airain précieux que l'on aurait purifié au creuset, sa voix comme la voix des grandes eaux. (1:13-15; voir aussi 4:3, 7; 6:12-14)

Quand il voit la Bête, elle ressemble «à une panthère, avec les pattes comme celles d'un ours, et la gueule comme une gueule de lion» (13:2; voir aussi 13: 11). Et quand la Jérusalem nouvelle apparait, à son tour, elle « resplendit telle un pierre très précieuse, comme une pierre de jaspe cristallin» (21: 11).

Les mots ne parviennent pas à rendre ce qui est vu. Ils sont en quelque sorte déficitaires et requièrent des substituts pour faire comprendre ce qu'il en est des phénomènes en présence. Le recours constant à la comparaison indique une intensification sémiotique soutenue, par la voie d'un enchaînement des interprétants. Car les «comme» et les «pareil» viennent témoigner de l'impossibilité de dire exactement, littéralement ce que c'est, et de la nécessité de recourir à d'autres signes, dont le rôle est de combler ce vide, de rendre présent malgré tout l'objet immédiat, et de le faire exister en pensée par substitution. La recherche de comparants est une sémiotisation. On s'éloigne de l'objet du monde (l'objet dynamique de Peirce), qu'un premier signe aurait pu donner l'illusion de rejoindre, pour s'enfoncer plus avant dans le langage, dans le sémiotique par l'enchaînement d'interprétants et de sémioses.

En plus des gestes de communication et des comparaisons, l'intensification de l'activité sémiotique se manifeste aussi par l'importance accordée aux désignations, aux noms à la signification parfois codée, c'est-à-dire à des appellations symboliques (11: 8) qui maintiennent le texte dans une illisibilité relative, propre à faire partager au lecteur les effets de la désémiotisation en cours. Il n'y a qu'à penser au chiffre de la bête, le 666 qui est dit représenter un nom d'homme (13: 18), ou à l'étoile Absinthe (8: 10), à Appolyôn ou Abaddôn, l'Ange de l'abîme (9: 11), à Babylone la Grande, la mère des prostituées et des abominations de la terre $(17: 5)$.

Cette intensification est surdéterminée, finalement, par une présence soutenue de signes et d'énigmes, tantôt révélés, tantôt tenus secrets. «Un signe grandiose apparut au ciel: une Femme!» (12:1); «Puis un second signe apparut au ciel: un énorme dragon rouge-feu, à sept têtes et dix cornes» (12: 3); «Puis je vis dans le ciel encore un signe, grand et merveilleux: sept Anges, portant sept fléaux» (15: 1). Or un signe est identifié explicitement comme signe uniquement quand il n'est pas transparent ou fonctionnel, et que l'objet immédiat auquel il renvoie n'occupe plus l'esprit qui tente de le saisir. L'attention ne porte plus alors que sur le signe lui-même, sa matérialité, son statut de signe-chose. Désigner quelque chose comme signe, sans dire de quoi il est le signe, c'est indiquer que sa sémiotisation n'est pas complète. Que la situation est déficitaire et qu'il y a un manque qui doit être résolu.

\section{LE LIVRE-ALIMENT}

Dans l'Apocalypse, la matérialité de la langue conséquente d'une désémiotisation prend la forme d'un livre qui se mange. Comment comprendre ce rapport à l'alimentation? Comprendre que manger un objet entraine un savoir? 
Gérard Haddad a consacré un essai à cette figure particulière du livre mangé. Elle s'inscrit pour lui dans une question plus large: comment le langage vient-il à l'homme? Haddad la rattache à la tradition hébraïque des rites alimentaires (ceux de la cacheroute et, plus largement, de la Halacha). Dans ces rites, et le premier exemple que retient Haddad est le seder, repas qui précède le jour de l'An, «les mets du repas symbolique ne se trouvent là que pour leur nom, pour les phonèmes qu'ils contiennent. [...] Ils sont le support de sons, de signifiants» (1984: 63). À ce repas, huit mets sont servis, faits de blette, de poireau, etc. Les membres de la famille en avalent une petite quantité après avoir énoncé une formule rituelle. Les mets apparaissent comme des alimentssymboles, où chaque denrée, par la voie d'homophonies aux noms hébraïques des végétaux utilisés, est identifiée à un mot ou un syntagme d'une proposition énoncée sous forme d'un vœu. Haddad explique:

Un premier vœu [...] consiste à souhaiter «que disparaissent tes ennemis et ceux qui nous veulent du mal». Le mot "disparaissent» de cette sentence, avant de manger de la blette, se dit en hébreu ystalekou, du verbe silek. On notera immédiatement l'homophonie avec salk, la blette.

Dans les mêmes conditions on énonce "que soient retranchés tes ennemis", etc., soit en hébreu yekaretou, du verbe karet, en homophonie avec krati, le poireau. (1984: 62)

Il apparaît ainsi, par l'utilisation de mots de même racine, que l'aliment est consommé pour sa valeur phonétique, pour ce jeu de correspondance que son nom permet de réaliser. «L'aliment devient une lettre», nous dit Haddad (1984: 63). D'ailleurs, cette association des mots aux aliments est une donnée majeure du ritualisme juif. Elle permet en fait d'effectuer une identification fondamentale: "On s'identifie à Dieu en incorporant cette "partie" qui lui coexiste de toute éternité, par qui et dans laquelle l'action divine se déploie: le Verbe, la Loi, la Torah" (1984: 71).

Manger le livre s'inscrit dans cette filiation d'un aliment devenu mot, inversé en mot devenu aliment, et qui marque l'identification, l'élection. Manger est un acte de langage, mais un acte d'une portée inhabituelle. Car partager le Verbe, l'incorporer, c'est connaitre Dieu en circonvenant les situations usuelles de communication, en ramenant la langue à sa matérialité, privilégiant non pas le sémantique, représenté traditionnellement par les relations établies entre les representamens et leurs objets de pensée, mais le médiologique (Debray, 1991), les liens nécessaires entre le representamen et ce qui le porte, le matériau qui en assure l'existence. C'est renverser, ne serait-ce que par un geste ponctuel, l'arbitrarité de la langue devenue effective avec la chute de la tour de Babel. Et dans l'Apocalypse, ce renversement permet d'atteindre l'extrême limite du sémiotique, là où les relations sont maintenues indépendamment des éléments en présence. Parler la langue de Dieu, se mettre sur le territoire ouvert par sa parole et son Verbe, c'est user d'interprétants qui procèdent aux attributions requises quels que soient les representamens utilisés. Des interprétants finals d'une telle force qu'ils rendent obsolète toute expérience première du signe, façon de neutraliser le sémiotique. Parler sans que les signes utilisés aient une quelconque importance, du fait d'être interchangeables, d'être des variables quand les objets immédiats auxquels il renvoient restent constants, c'est échapper aux contraintes du sémiotique.

Dans les termes plus précis de la sémiotique peircienne, on se retrouve en effet, ou en deçà de l'interprétant immédiat, celui qui est révélé dans la compréhension correcte du signe lui-même, ou audelà de l'interprétant final troisième, qui est l'interprétant systématique par excellence, décisoirement déductif. Nous sommes donc en présence, ou d'une sémiose rabattue à ses representamens clivés des renvois qui les constituent comme signes, puisque les interprétants ne sont pas là pour procéder aux attributions; ou d'une sémiose qui ne requiert plus la présence de signes pour perdurer, les attributions étant maintenues malgré l'interchangeabilité des representamens. Deux situations limites où se trouble le rapport au sémiotique? . 
Ce pas, d'ailleurs, il est franchi non pas dans la scène fondatrice de la prophétie d'Ézéchiel, mais dans l'Apocalypse de Jean, et il est fait à la lumière du miracle de la Pentecôte. Haddad indique qu'une différence essentielle entre les scènes d'Ézéchiel et de Jean est l'apparition chez ce dernier de l'amertume du livre. Et il se demande de façon fort pertinente si cette absence d'amertume ne reflète pas «la position différente du judaïsme par rapport au livre» (1984: 104)? Mais une autre différence est tout aussi essentielle et elle concerne les effets de cette ingestion. Chez Ézéchiel, cette maîtrise de la langue et de la parole de Dieu n'est pas transcendante: «Ce n'est pas vers un peuple au parler obscur et à la langue difficile que tu es envoyé, c'est vers la maison d'Israël» (Ézé, 3:5). Nous restons dans du sémiotique, dans le cadre restreint d'une langue déjà connue, qui sert de dénominateur commun. Il y a bien une ouverture faite à des peuples aux langues obscures et difficiles («si je t'envoyais vers eux, ils t'écouteraient» [Ézé, 3:6]), mais elle n'est qu'une possibilité, identifiée sans être directement impliquée. Tout autre est la situation de Jean qui, après avoir mangé le livre, doit aller "prophétiser contre une foule de peuples, de nations, de langues et de rois» (Apo, 10: 11). La parole de Dieu ouvre la voie à une sursémiotisation (ou plus simplement à une désémiotisation par le haut). Cette dynamique particulière apparaît donc dans le monde chrétien. Elle survient en fait dans une eschatologie où sont séparés le bien et le mal, le haut et le bas, l'ascension des uns qui sauront se défaire de leur enveloppe charnelle, l'enfouissement des autres qui ne seront plus qu'un corps douloureux. Et elle en reproduit le mouvement à même le langage.

\section{DE LA PIERRE ET DES MOTS}

J'ai fait l'hypothèse que la désémiotisation était une marque de l'imaginaire de la fin et j'en ai décrit le processus en fonction d'un imaginaire religieux et eschatologique, celui de l'Apocalypse, mais aussi, à un degré moindre, celui du Dernier mot de Blanchot, qui se déploie à partir d'un intertexte biblique. Dans les deux cas, le processus a pris une double forme, dégradation d'une langue qui s'opacifie, et dont le stade ultime est une inquiétante matérialité, une langue lourde comme de la pierre; passage à un état où le sémiotique n'est plus nécessaire, pour cause de transcendance. J'aimerais montrer, maintenant, que la portée de cette hypothèse déborde du cadre religieux, que la désémiotisation est une marque de toute pensée de la fin, d'un imaginaire du moins qui ne se contente pas de mettre en scène un répertoire d'incidents et d'actions facilement stéréotypés, un bestiaire déjà éprouvé, mais qui tente de penser les effets intimes et complexes d'une expérience de la fin et de ses frontières. Pour le montrer, en guise de conclusion, je décrirai la scène finale de City of Glass, premier des romans de la trilogie new-yorkaise de Paul Auster (1990). Entre autres, parce que la situation sémiotique qu'on y retrouve reprend cette polarité du processus de désémiotisation, dans un contexte qui n'est pas apocalyptique.

Roman postmoderne, dérive sur le genre policier, réflexion sur les identités narratives, et encore sur les limites du langage et de ses possibilités de représentation, City of Glass fait de la chute de la Tour de Babel et de la récupération d'une langue adamique l'un de ses enjeux. Après une longue enquête qui le mène au bord de l'anéantissement, Quinn, le personnage principal, retourne dans l'appartement maintenant vide de son client. Tout au long de son travail, il a noté dans un carnet rouge ses propres faits et gestes, ses hypothèses sur l'homme qu'il a suivi avec diligence. Il s'isole dans l'appartement, se défait de ses vêtements et entreprend de poursuivre une écriture qui aboutira à sa propre disparition.

Installé tel un ascète dans une pièce où il fait de plus en plus sombre, où les journées semblent raccourcir indéfiniment, il se consacre entièrement à son carnet. Le long processus de son enquête n'a pas eu pour résultat d'arrêter un coupable mais de faire perdre à Quinn une fois pour toutes son identité. Son cahier rouge est son dernier lien, son dernier trait d'existence (Gervais, 1995). Et, dans un moment paradoxal d'une grande étrangeté, quand son carnet rouge est rempli, Quinn ne fait pas qu'arrêter d'écrire, 
il s'évapore littéralement. Quand sa dernière phrase est écrite, les derniers mots tracés en bas de la dernière page, plus rien de lui ne subsiste. Voici les derniers moments de la narration:

This period of growing darkness coincided with the dwindling of pages in the red notebook. Little by little, Quinn was coming to the end. At a certain point, he realized that the more he wrote, the sooner the time would come when he could no longer write anything. [...] Quinn no longer had any interest in himself. He wrote about the stars, the earth, his hopes for mankind. He felt that his words had been severed from him, that now they were a part of the world at large, as real and specific as a stone, or a lake, or a flower. They no longer had anything to do with him. He remembered the moment of his birth and how he had been pulled gently from his mother's womb. He remembered the infinite kindness of the world and all the people he had ever loved. Nothing mattered now but the beauty of all this. He wanted to go on writing about it, and it pained him to know that this would not be possible. Nevertheless, he tried to face the end of the red notebook with courage. He wondered if he had it in him to write without a pen, if he could learn to speak instead, filling the darkness with his voice, speaking the words into the air, into the walls, into the city, even if the light never came back. The last sentence of the red notebook reads: "What will happen when there are no more pages in the red notebook?»

(1990: 156-7)

L'écrit de Quinn s'arrête sur cette dernière phrase. La perspective change, aussitôt la question posée - et à laquelle il incombe au lecteur de répondre -, et un narrateur anonyme nous apprend qu'aucune trace de Quinn n'a été retrouvée. Le carnet rouge est découvert, abandonné sur le sol de l'appartement, mais Quinn a disparu. Dans la logique du roman, compte tenu des liens qui sont établis de façon utopique entre les mots et les choses, de cette motivation adamique du langage, tout suggère que Quinn n'est pas simplement sorti de l'appartement, une fois les pages du carnet remplies, mais qu'il s'est évanoui, volatilisé, dans une ascension inédite. Il n'était plus que langage, qu'une écriture et, sans elle, il n'existe plus. La fin du carnet marque sa propre fin. Il n'a plus de raison d'être; par conséquent, il n'est plus.
Une telle interprétation corrobore l'hypothèse du double processus de désémiotisation à l'œuvre à l'approche de la fin, d'une instabilité des interprétants comme manifestation d'un imaginaire de la fin. Cette dernière ne passe pas par un excès d'événements et de personnages, mais par une épuisement graduel du monde, et du carnet. Quinn se dévêt, retournant à un état de pureté originel, il s'enferme dans une chambre vide, au fond d'un appartement vide, et il se détache au fur et à mesure du monde. Ses derniers propos ne portent plus sur l'enquête, sur les derniers fils qu'il n'a pas réussi à relier, mais sur des souvenirs. Il retourne à un état d'innocence, celui qui prévalait à sa naissance, offerte comme mise en abyme du processus qui suivra, une autre naissance, mais hors du monde et de sa matérialité. City of Glass est un texte de l'épuisement, celui bien réel d'un personnage qui ne se laisse pas mourir, mais choisit plutôt de se rendre à la limite de la vie et de la parole, là où les deux s'emmêlent et se complètent comme les faces d'une bande de Moebius. Et, à ce moment ultime de la fin, le processus de désémiotisation se déploie dans toute sa force. Le langage devient chose pour lui, les mots se détachent et commencent à exister indépendamment de l'énonciateur, mis au monde et aussi réels qu'un lac, qu'une fleur, qu'une pierre. Des mots, redevenus choses, comme de la pierre ${ }^{10}$. Mais en même temps, s'ouvre l'accès à un langage transcendant, à un mode langagier qui ne requiert plus de signes en tant que tel, libéré de toute matérialité. Le personnage souhaite avoir accès à une écriture sans crayon, une écriture qui serait parole, mais sans marque pour la faire exister, sans énonciation pour la transmettre. Une parole pure, sursémiotisée puisque dégagée de la nécessité d'une écriture, d'un système de traces. De la pierre en guise de mots et une écriture sans graphie. Une langue dépossédée de toute subjectivité, d'une part, un idéal antérieur au langage, de l'autre.

Nous retrouvons les deux limites de la désémiotisation des imaginaires de la fin. Le dernier mot, le livre mangé et le carnet rouge, s'ils répondent à des logiques fictionnelles distinctes, recouvrent une 
même réalité sémiotique, un même processus de perte.

Cette régularité incite en fait à poser la désémiotisation comme un trait essentiel de l'imaginaire de la fin. Comme une de ses marques constitutives. Les exemples déployés ici ont servi à poser l'hypothèse. Il reste encore à l'étayer. On en trouve des traces évidentes dans les écrits d'Antonin Artaud et de Samuel Beckett, par exemple, et on en découvre aussi les mécanismes dans des romans tels La petite fille qui aimait trop les allumettes, de Gaétan Soucy (1998). Dans ce roman, les dysfonctionnements langagiers du «narrateur» apparaissent, à la lumière de l'hypothèse, comme les indices d'un imaginaire de la fin, d'autant plus marquant qu'il agit longtemps de façon tacite. Le couple opacité et fin y refait surface, selon une équation nouvelle. Il est tard pour s'étendre davantage sur cet exemple, mais sa seule présence permet de montrer que la régularité notée ici s'applique aisément à un corpus plus vaste, qu'elle peut agir non seulement comme hypothèse mais comme principe de lisibilité. La perte du langage comme bien d'autres déséquilibres langagiers semblent bel et bien s'inscrire aux limites de l'imagination, là où inévitablement l'imaginaire rejoint sa fin.

\section{N O TES}

1.J'ai repris ici sommairement l'analyse que j'ai faite de ce texte de Blanchot dans « Presbytère, hiéroglyphe et dernier mot. Pour une définition de l'illisibilité» (La Lecture littéraire, Reims, 1999). Cet article s'inscrit dans le cadre des activités de l'équipe de recherche de l'Imaginaire de la fin, subventionnée par les Fonds FCAR.

2.Sauf indication contraire, je me servirai de la traduction faite sous la direction de l'École biblique de Jérusalem, Paris, Éd. du Cerf, 1998.

3. Le personnage explique son geste en narguant ses interlocuteurs: «Écoute à présent ce que dit la voix: tiens secrètes les paroles des sept tonnerres et ne les écris pas; tiens, mange-le; il te remplira les entrailles d'amertume, mais en ta bouche il aura la douceur du miel. Tu vois? Maintenant je scelle ce qui ne devait pas être dit, dans la tombe que je deviens" (Eco, 1982: 485).

4.Une traduction comme celle des moines de Maredsous (1969) opte plutôt pour "une bande de papyrus qu'on enroule", tout en maintenant au chapitre dix l'idée d'un "petit livre ouvert", qui témoigne bel et bien de la coexistence de ces deux formes, dans le texte. Fanny et Gilles Deleuze, dans leur préface au commentaire de l'Apocalypse de D.H. Lawrence, se sont étonnés de cette indétermination: "Quand le livre est encore un rouleau, peut-être garde-t-il une puissance de symbole. Mais justement comment expliquer cette bizarrerie, que le livre des sept sceaux est censé être un rouleau, et pourtant que les sceaux soient brisés successivement, par étapes, tant L'Apocalypse a besoin de mettre des points partout, d'installer des segments partout?» (Lawrence, 1978 (1931): 31).

5. On retrouve par contre l'amertume quelques versets plus loin, quand Ézéchiel explique: «Et l'Esprit m'enleva et me prit; j'allai amer, l'esprit enfiévré, et la main de Yahvé pesait fortement sur moi" (3:14). 6.Il faut noter par contre l'ajout que fait Pierre à la prophétie de Joël, car le texte dit plus simplement: «Après cela je répandrai mon Esprit sur toute chair" (Joël 3:1). La mention des "derniers jours " apparaît dans les Actes des Apôtres.

7. «Celui qui a des oreilles, qu'il entende ce que l'Esprit dit aux Églises ", apprend-il dès le début de ses visions (la même formule est répétée six fois, puis reprise en 13:9).

8. Les prépositions sont d'ailleurs nombreuses à assurer une continué aux divers moments de l'Apocalypse. Les puis, après, ensuite, alors, et, encore, ainsi, voici, lorsque, et les locutions prépositives afférentes se succèdent pour lier les événements, les ordonner en propositions narratives.

9. Dans une telle conception, le sémiotique n'est pas un idéal, un état qui est maintenu quoi qu'il arrive, mais une situation de fait, pragmatique, qui a ses limites et qui peut connaître des ratés et des transformations.

10. Cette analogie est un leitmotiv de la première écriture de Paul Auster. Dans sa poésie (notamment le cycle des Disappearances [Ground Work, 1990]), mais aussi dans ses romans jusqu'à The Music of Chance, l'opacité de la langue est associée à la pierre, à un mur de pierre, contre lequel la conscience ne cesse de buter. 


\section{RÉFÉRENCES BIBLIO G RAPH IQ U ES}

Apocalypse de Jean [1993]: trad. et notes de Claude Tresmontant, Paris, F.X. de Guibert.

La Bible [1998]: trad. sous la dir. de l'École biblique de Jérusalem, Paris Éd. du Cerf;

[1969]: version établie par les moines de Maredsous, ParisTurnhout, Brepols.

Auster, P. [1990]: The New York Trilogy, , New York, Penguin Books; [1990]: Ground Work, Londres, Faber \& Faber. Blanchot, M. [1983] : Après coup, Paris, Minuit.

Cavallo, G. et R. Chartier (sous la dir. de) [1997] : Histoire de la lecture dans le monde occidental, Paris, Seuil.

CHARTIER, R. (sous la dir. de) [1995]: Histoire de la lecture; un bilan des recherches, Paris, IMEC, Éd. de la Maison des sciences de l'homme. ChouraQui, A. [1982] : L'Univers de la Bible, Paris, Lidis, vol. 9. DEBRAY, R. [1991]: Cours de médiologie générale, Paris, Gallimard. Deledalle, G. [1979]: Théorie et pratique du signe, Paris, Payot. ECO, U. [1992]: Les Limites de l'interprétation, Paris, Grasset; [1982]: Le Nom de la rose, Paris, Grasset.

GervaIs, B. [1995]: "Au pays des tout derniers mots : une Cité de verre aux limites du langage», L'CEuvre de Paul Auster, Actes sud/Université de Provence, 86-101;

[1999]: «Presbytère, hiéroglyphes et dernier mot. Pour une définition de l'illisibilité», La lecture littéraire, Paris, Klincksieck, n³, janvier, 205-228.

HADDAD, G. [1984]: Manger le livre, Paris, Grasset.

JuNGER, E. [1998] : Sertissages à propos de l'Apocalypse, Cognac, Fata Morgana.

KERMODE, F. [1966]: The Sense of an Ending, Oxford, Oxford U.P.

LAWRENCE, D.H. [1931] : Apocalypse, Paris, Balland (1978).

MÂLE, É. [1925]: L'Art religieux de la fin du Moyen-Âge en France, Paris, Librairie Armand Colin.

Manguel, A. [1996]: A History of Reading, Toronto, Alfred A. Knopf Canada.

PeIRCE, C. S. [1978] : Ecrits sur le signe, Paris, Seuil.

SAENGER, P. [1982] : "Silent Reading: its Impact on Late Medevial

Script and Society", Viator, vol. 13, 367-414.

SoUCY, G., [1998] : La petite fille qui aimait trop les allumettes, Montréal, Boréal.

STEINER, G. [1969]: Langage et Silence, Paris, Seuil.

Tresmontant, C. [1984] : Apocalypse de Jean, Paris, F.X. de Guibert. 